\title{
Integrated-Optic Electric-Field Sensor Utilizing a Ti:LiNbO 3 Y-fed Balanced-Bridge Mach-Zehnder Interferometric Modulator With a Segmented Dipole Antenna
}

\author{
Hongsik Jung* \\ Department of Electronic and Electrical Engineering, Hongik University, \\ 2639, Sejong-ro, Jochiwon, Sejong 339-701, Korea
}

(Received August 7, 2014 : revised November 20, 2014 : accepted November 20, 2014)

\begin{abstract}
We have demonstrated a $\mathrm{Ti}: \mathrm{LiNbO}_{3}$ electro-optic electric-field sensor utilizing a $1 \times 2 \mathrm{Y}$-fed balanced-bridge Mach-Zehnder interferometric (YBB-MZI) modulator, which uses a 3-dB directional coupler at the output and has two complementary output waveguides. A dc switching voltage of $\sim 25 \mathrm{~V}$ and an extinction ratio of $\sim 12.5 \mathrm{~dB}$ are observed at a wavelength of $1.3 \mu \mathrm{m}$. For a $20 \mathrm{dBm}$ rf input power, the minimum detectable electric fields are $\sim 8.21,7.24$, and $\sim 13.3 \mathrm{~V} / \mathrm{m}$, corresponding to dynamic ranges of $\sim 10, \sim 12$, and $\sim 7 \mathrm{~dB}$ at frequencies of 10,30, and $50 \mathrm{MHz}$ respectively. The sensors exhibit almost linear response for an applied electric-field intensity from $0.29 \mathrm{~V} / \mathrm{m}$ to $29.8 \mathrm{~V} / \mathrm{m}$.
\end{abstract}

\begin{abstract}
Keywords : Electro-optical electric-field sensor, $\mathrm{Ti}$ : $\mathrm{LiNbO}_{3}$ optical channel waveguide, Lithium niobate, Y-fed balanced-bridge Mach-Zehnder interferometric optical modulator, Segmented dipole antenna

OCIS codes : (130.3120) Integrated optic devices; (130.3730) Lithium niobate; (230.2090) Electro-optical devices; (230.7380) Channel waveguides; (280.4778) Optical sensing and sensors
\end{abstract}

\section{INTRODUCTION}

Electric-field sensors with broad, flat frequency responses are important tools for electromagnetic compatibility and interference $(\mathrm{EMC} / \mathrm{EMI})$ measurements, high-frequency electronic circuit diagnostics, medical equipment field monitoring, radiofrequency reception, and high-power microwave detection, and their importance is increasing with the progress in mobile multimedia communications $[1,2]$. To evaluate EMC, it is necessary to accurately evaluate the strength and distribution of the electromagnetic field surrounding the electronic equipment. Based on the potential applications mentioned above, the requirements for electric-field sensors are as follows: wide frequency bandwidth and large dynamic range; high spatial resolution and little interference with the original field; and high stability and accuracy.

Even though various kinds of sensing modules have been developed, those with photonic links reduce or eliminate some of the inaccuracies and systematic errors that affect measurement techniques using conventional EM-field sensors. They provide electrical isolation, which eliminates ground loops and common-mode electrical pickup between the sensor head and the electronics module. The optical fibers and dielectric components produce minimal field distortion. In addition, they can preserve both the phase and amplitude of high-frequency fields, with good fidelity and low losses. The development of optical-fiber and optoelectronic components for the telecommunications industry has made it possible to implement photonic sensors that are accurate and convenient to use.

In particular, titanium-diffused lithium niobate $\left(\mathrm{Ti}: \mathrm{LiNbO}_{3}\right)$ waveguide devices are suitable for electric-field detection, since the sensor will not perturb the field to be measured. A linear modulator that is passively biased at the optimum linear operating point is desired. This has been demonstrated in Mach-Zehnder interferometers $(\mathrm{MZI})$ and $1 \times 2$ directional couplers. The former devices need an intrinsic bias of $\pi / 2$, where a geometrical path length difference of a quarter of a wavelength is required between the two arms. [3-5] However, it is not easy to obtain the proper operating point through only the path length difference, because of fabrication tolerances. The latter devices are automatically biased at the optimum $3-\mathrm{dB}$ operating point by symmetry of design, offering the possibility of a more fabrication-tolerant device

\footnotetext{
*Corresponding author: hsjung@wow.hongik.ac.kr

Color versions of one or more of the figures in this paper are available online.
} 
than the asymmetric Mach-Zehnder interferometric modulators. [6-8] However, the transfer function is complicated, and the sensor performance will be unacceptable for a certain value (namely the ratio of interaction length to conversion length). [8]

In contrast, a Y-fed balanced-bridge MZI modulator (YBBMZI) uses a 3-dB coupler at the output and has two complementary output waveguides. [9-11] It has a well-defined transfer function for the output optical power versus sensed electric-field intensity, and can be automatically biased at the optimum 3-dB operating point by symmetry of design, offering the possibility of a more fabrication-tolerant device than either an asymmetric Mach-Zehnder interferometric modulator or an $1 \times 2$ directional coupler. A mono-shield gold electrode structure was applied in a YBB-MZI by the Tsinghua University group to detect very high electricfield. [9] In general, the minimum detectable electric-field strength is limited by the relative intensity noise (RIN) of the laser diode. Therefore, the YBB-MZI configuration has been proposed for reducing RIN and improving sensitivity with a balanced optical receiver by a German group. [11]

In this paper, we provide the quantitative theory of a YBB-MZI modulator and report on a fabricated $\mathrm{Ti}: \mathrm{LiNbO}_{3}$ YBB-MZI modulator operating at a wavelength of $1.3 \mu \mathrm{m}$. Also, we present in detail the fabrication and design parameters as well as the optical and electrical performances of a packaged electric-field sensor with a segmented dipole patch antenna, including minimum detectable electric-field intensity, dynamic range, and sensitivity.

\section{THEORY, FABRICATION, AND PERFORMANCE OF A Ti:LiNbO 3 1×2 YBB-MZI MODULATOR}

\subsection{Device Theory}

The YBB-MZI modulator consists of a 3-dB directional coupler at the output and has two complementary output waveguides, as shown in Fig. 1. For electric-field sensing, a segmented dipole antenna has been arranged between two arms of the MZI structure.

A $2 \times 2$ directional coupler is characterized by the coupling length $L_{c}$, the coupling coefficient $\kappa$, and the wavenumber $\beta$ of the waveguide. Ignoring transmission loss, the transfer matrix of a directional coupler is given by [12]

$$
\left(\frac{\overline{E_{01}}}{\overline{E_{02}}}\right)=e^{-i \beta L_{c}}\left(\begin{array}{cc}
\cos \kappa L_{c} & -j \sin \kappa L_{c} \\
-j \sin \kappa L_{c} & \cos \kappa L_{c}
\end{array}\right)\left(\frac{\overline{E_{l 1}}}{\overline{E_{l 2}}}\right)
$$

The incident single-mode light is equally divided in two by the input $3-\mathrm{dB}$ splitter and can be described as follows:

$$
\overline{E_{i 1}}, \overline{E_{i 2}}=\frac{1}{\sqrt{2}} e^{-j} \theta
$$

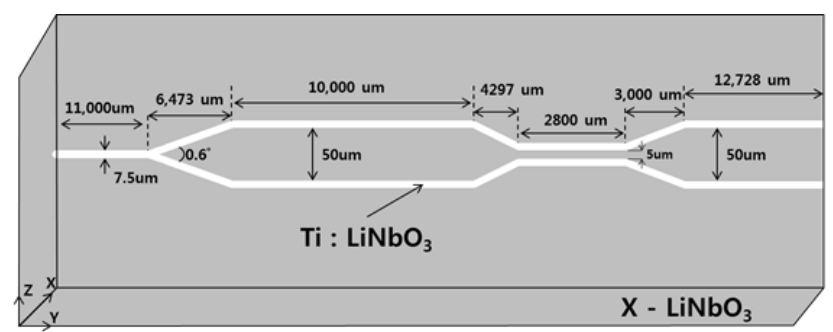

(a)

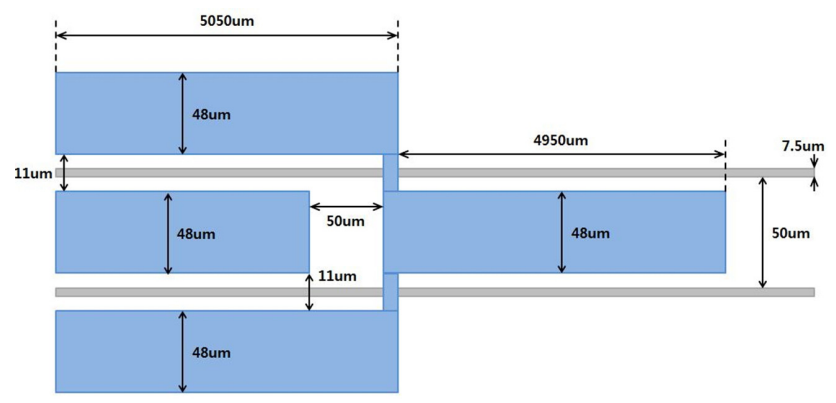

(b)

FIG. 1. Schematic diagrams and dimensions of (a) $\mathrm{Ti}_{\mathrm{LiNbO}}$ $1 \times 2$ YBB-MZI modulator and (b) segmented dipole patch antenna.

where $\theta$ is the initial phase.

The segmented dipole patch antenna with an electrode induces the electric field on the parallel two arms of the MZI, which results in a change of refractive index and an unbalanced modulation, as shown in Fig. 1. Before entering the output directional coupler, the light in the two arms has an extrinsic phase mismatch $\phi\left(E_{e}\right)$ due to the sensed electric field. The phase mismatch $\phi\left(E_{e}\right)$ can be written as

$$
\phi\left(E_{e}\right)= \pm \frac{\pi}{\lambda} n_{e}^{3} \gamma_{33} \Pi_{e} E_{e}
$$

where $l_{e}$ is the length of the electrode connected to the segmented dipole patch antenna, $r_{33}$ is the electro-optic coefficient of lithium niobate $(\sim 30 \mathrm{pm} / \mathrm{V}), \lambda$ is the optical wavelength, $n_{e}$ is the extraordinary refractive index of lithium niobate, $E_{e}$ is the electric field intensity inside the waveguide, and $\Gamma(0<\Gamma<1)$ is the overlap integral between the applied electrical field and the optical field. Therefore, the light entering the output coupler can be expressed as

$$
\begin{aligned}
& \overline{E_{i 1}}=\frac{1}{\sqrt{2}} e^{-j\left(\theta+\phi\left(E_{e}\right)\right)} \\
& \overline{E_{i 2}}=\frac{1}{\sqrt{2}} e^{-j \theta}
\end{aligned}
$$

Combining (1) with (4), the output power of the YBBMZI modulator is derived as 


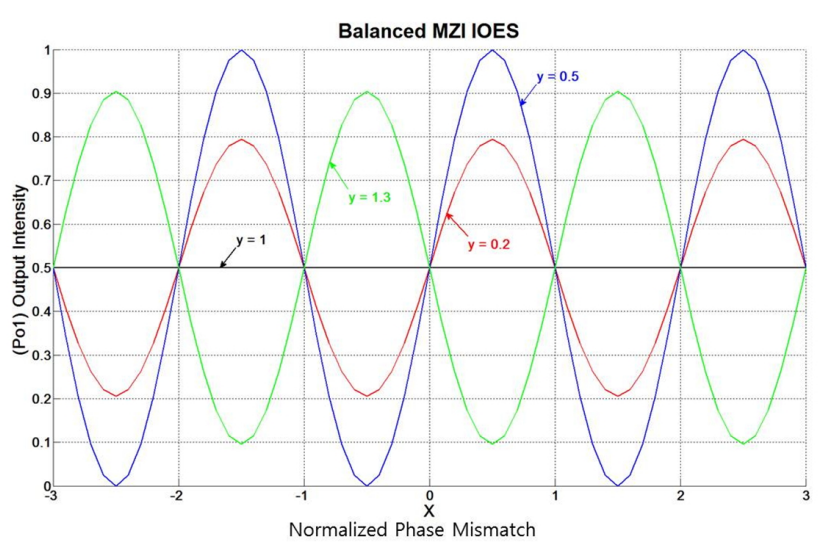

FIG. 2. Simulation results for the light output intensity versus driving voltage with $\mathrm{y}=0.2,0.5,1$, and 1.3 .

$$
\begin{aligned}
P_{01} & =\frac{1}{2}[1+\sin (\pi y) \cdot \sin (\pi x)] \\
& =\frac{1}{2}\left[1+\sin \left(2 k L_{c}\right) \cdot \sin \left(\phi\left(E_{e}\right)\right)\right] \\
P_{02} & =\frac{1}{2}[1-\sin (\pi y) \cdot \sin (\pi x)] \\
& =\frac{1}{2}\left[1-\sin \left(2 k L_{c}\right) \cdot \sin \left(\phi\left(E_{e}\right)\right)\right]
\end{aligned}
$$

where $x=\phi\left(E_{e}\right) / \pi$ is the normalized phase mismatch, $y=L_{c} / l_{c}$ is the normalized coupling length, and $l_{c}=\pi / 2 k$ is the coupling conversion length.

The output intensity $P_{o l}$ is calculated and plotted for the YBB-MZI electric-field sensor, as shown in Fig. 2. The YBB-MZI sensor has a sinusoidal transfer function for different $y$ values. The value of $y$ only affects the extinction ratio, which can be expressed as $\sin (\pi y)$. For most cases (where $\sin (\pi y) \neq 0$ ) the transfer function is acceptable, as the extinction ratio only impacts the $E$-field measurement sensitivity. In order to guarantee the best sensitivity, the coupling length should satisfy the condition

$$
\begin{aligned}
& \sin \left(2 k \cdot L_{c}\right)=1 \\
& k \cdot L_{c}=\frac{(2 n+1) \pi}{4}, n=0,1,2 \ldots
\end{aligned}
$$

\subsection{Design and Fabrication}

A single-mode channel waveguide symmetric, $1 \times 2 \mathrm{Y}$-fed Balanced-Bridge Mach-Zehnder Interferometric Modulator with a segmented dipole patch antenna was designed for operation at a wavelength of $\sim 1.3 \mu \mathrm{m}$ in $x$-cut, $y$-propagating $\mathrm{LiNbO}_{3}$ substrate, as shown in Fig. 1. The device consists of a Y-branch splitter, a phase modulator, and a directional coupler. The overall device structure is similar to that of a Mach-Zehnder interferometer with two output ports. The waveguide width is $7.5 \mu \mathrm{m}$, and the splitting angle of the Y-branch is $0.6^{\circ}$. The gap width between two adjacent wave-

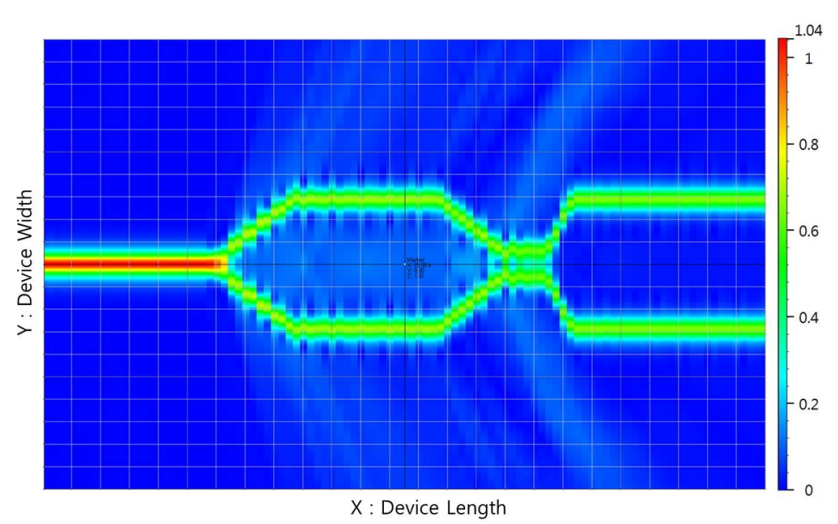

(a)

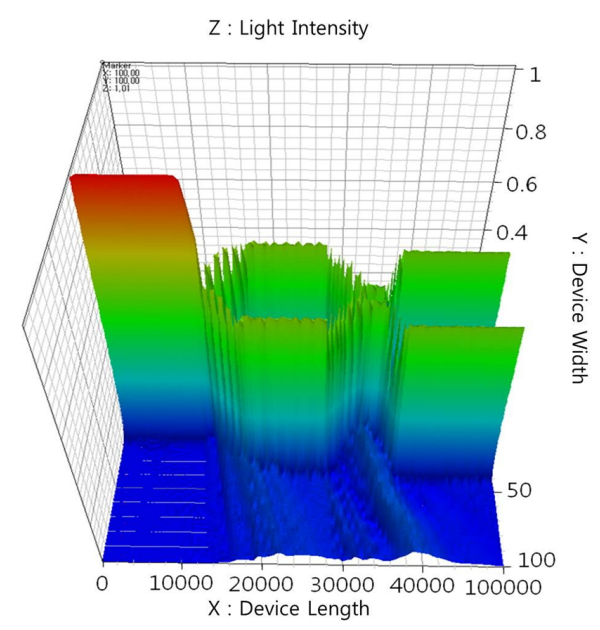

(b)

FIG. 3. (a) Two-dimensional and (b) three-dimensional BPM-CAD simulation results.

guides of the directional coupler is $5 \mu \mathrm{m}$, and the parallel coupling length $L_{c}$ is $2.8 \mathrm{~mm}$. The distance between the inner edges of the two output waveguides is $50 \mu \mathrm{m}$. The length and gap of the modulation electrode connected to the dipole patch antenna are $5 \mathrm{~mm}$ and $11 \mu \mathrm{m}$ respectively, as shown in Fig. 1(b). The BPM-CAD two- and three-dimensional simulation results of light-wave propagation through the YBBMZI modulator are shown in Fig. 3 [13]. It is clearly seen that the incident light splits almost equally at the input Y-branch and directional coupler, respectively. The dimensions and arrangement of the segmented dipole patch antenna with a lumped electrode are also shown in Fig. 1(b).

The $1 \times 2$ YBB-MZI modulators were fabricated on $x$-cut $\mathrm{LiNbO}_{3}$ substrate using standard photolithography. Channel waveguides were formed by diffusing a strip of $\mathrm{Ti}$ film $1050 \AA$ thick and $7.5 \mu \mathrm{m}$ wide at $1050^{\circ} \mathrm{C}$ in wet ambient. The substrate edges were optically polished to allow buttcoupling and pig-tailing. An $\mathrm{SiO}_{2}$ buffer layer $\sim 3000 \AA$ thick was deposited using e-beam evaporation and $99.99 \%$ pure $\mathrm{SiO}_{2}$ pellets, to prevent propagation loss due to the optical absorption of the antenna metal. An aluminum segmented dipole antenna electrode $\sim 5000 \AA$ thick was fabricated to 


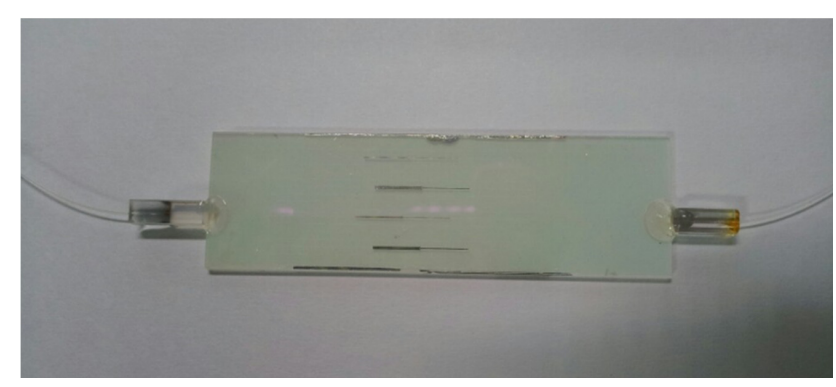

FIG. 4. Photograph of the fabricated device with pig-tailed optical fibers.

allow the sensing of the electric field. Polarization-maintaining single-mode and multimode optical fibers were attached to the input and output waveguides respectively. Figure 4 shows the micrograph of the fabricated device, with attached optical fibers and a segmented dipole patch antenna. The measured insertion loss of the device with input/output fiber was about $8.33 \mathrm{~dB}$, which includes fiber-connector loss, pig-tailing loss, mode-mismatch loss, and propagation loss.

\subsection{Evaluation of Performance}

We first tested the devices without attached fibers by applying dc voltage. Performance characterization was carried out at a wavelength of $1.3 \mu \mathrm{m}$ by butt-coupling the device with a pigtailed tunable laser. TE polarized light from the pigtailed tunable laser was butt-coupled to the devices, collected at the output by a microscope objective, and focused onto a photodetector for characterization. TE or TM input polarization was selected by proper adjustment of a fiber polarization controller. Single-mode propagation for TE polarization was observed in the $1 \times 2$ YBB-MZI modulator.

When no voltage was applied, the optical output powers of the two branches were almost identical. Therefore, the modulator was intrinsically set at the $\sim 3$-dB half-point. When the driving voltage was increased or decreased to $\pm \sim 12.5$ $\mathrm{V}$, the light in one branch of YBB-MZI modulator was coupled to the other branch, where the light output power of the former branch disappeared and the output of the latter reached its maximum. It was observed that the switching voltage applied to modulate the light power of either branch from a bar state (maximum intensity) to a cross state (minimum intensity) was $\sim 25 \mathrm{~V}$, corresponding to a light-extinction ratio of $12.5 \mathrm{~dB}$. Figure 5 shows the optical output power versus applied voltage, measured by an optical power meter. It exhibits a slightly asymmetric dc output characteristic curve and a switching voltage of $\sim 25 \mathrm{~V}$. The ac modulation responses of the two branches versus driving voltage are further presented in Fig. 6. The overlapping, smaller sinusoidal curve is the optical signal, while the bigger curve is the applied ac voltage signal at a frequency of $\sim 1 \mathrm{kHz}(5 \mathrm{~V} /$ div). The output power levels of the two ports are almost the same, and the sinusoidal curves also show a good inverse relation, due to the power being transferred periodically between the two ports.

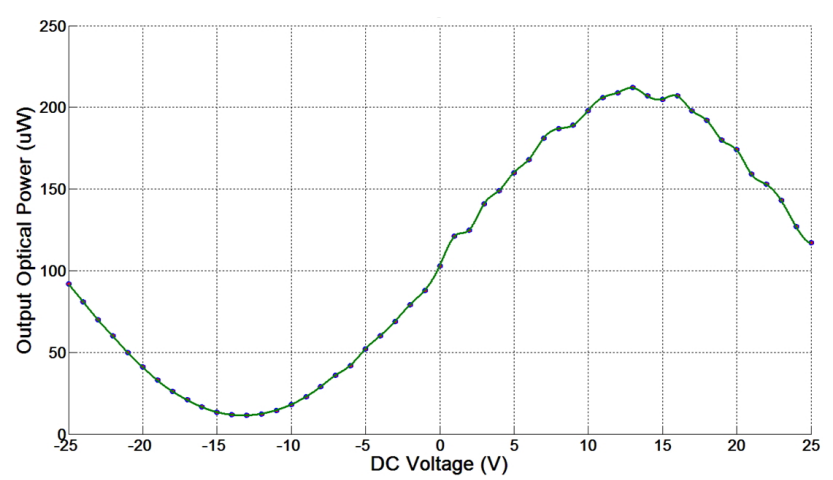

FIG. 5. Measured optical output power intensity versus applied dc voltage.

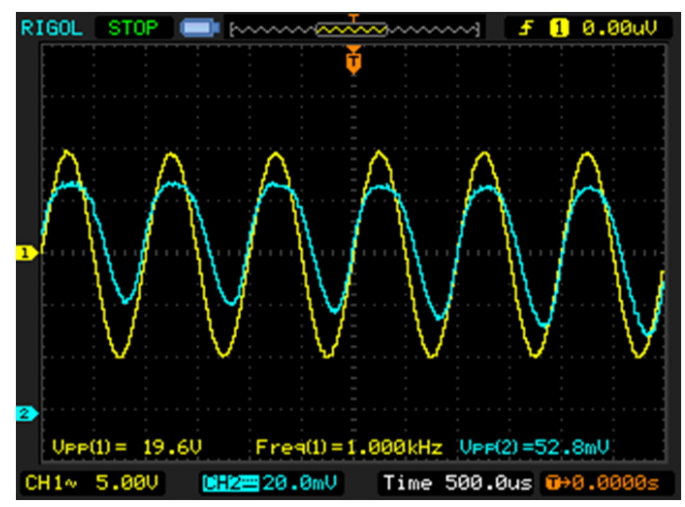

(a)

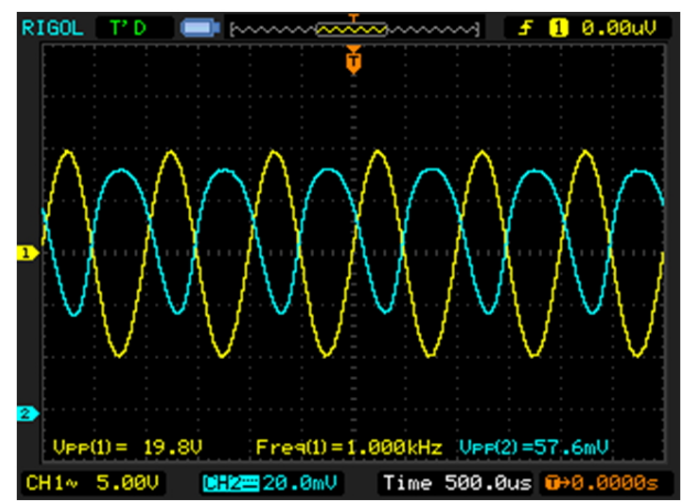

(b)

FIG. 6. The 1-kHz ac modulation responses at the (a) upper and (b) lower output port, as shown in Fig. 1(a).

\section{MEASUREMENT AND EXPERIMENTAL RESULTS}

\subsection{Experimental Set-Up}

Frequency tests, to evaluate the minimum detectable field and frequency response of the fiber-coupled sensor, were performed using a tunable laser operating at a wavelength of $1.3 \mu \mathrm{m}$. The incident optical power was of the order of $1.4 \mathrm{~mW}$. Figure 7 shows a block diagram of the experi- 


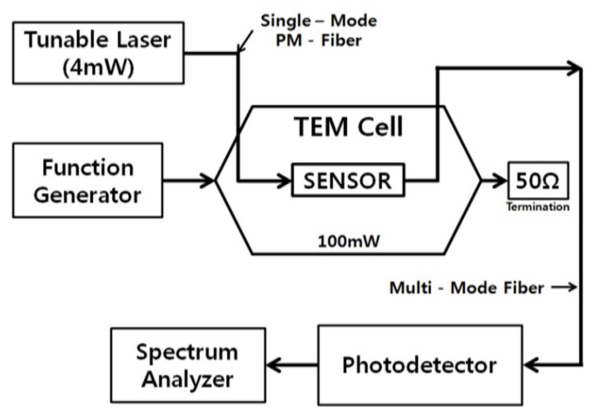

FIG. 7. Block diagram of the test setup for measurement of electric-field sensing and evaluation of frequency response.

ment setup. The device was tested in a uniform electric field by placing it in a Transverse Electro Magnetic (TEM) cell (Tescom TC-5010A), as indicated in Fig. 7. The TEM cell is used to generate accurate electro-magnetic (EM) waves over a wide frequency range. EM waves generated in the cell propagate in the transverse mode and have the same characteristics as plane waves. The optical fibers are fed through the sloping walls of the TEM cell and connected to the laser and photodetector via $\mathrm{FC} / \mathrm{PC}$ fiber-optic connectors. The applied electric-field strength was calculated from the output level and the dimensions of the TEM cell. The -20 $-+20 \mathrm{dBm}(10 \mu \mathrm{W}-100 \mu \mathrm{mW})$ rf input to the TEM cell corresponds to electric-field strength from $0.293 \mathrm{~V} / \mathrm{m}$ to $23.2 \mathrm{~V} / \mathrm{m}$. The actual field intensity experienced on the sensor substrate at $23.2 \mathrm{~V} / \mathrm{m}$ in TEM cell is $0.66 \mathrm{~V} / \mathrm{m}$, due to the high dielectric constant of $\mathrm{LiNbO}_{3}(\varepsilon \approx 35)$. The rf power travels through the TEM cell in the same direction as the propagation of light in the optical fibers and sensor. [7]

\subsection{Test Results and Discussion}

Figure 8 show the spectrum-analyzer outputs for input of $20 \mathrm{dBm}$ to the TEM cell at frequencies of 10,30 , and $50 \mathrm{MHz}$ respectively. The rf power received at the photodetector was measured as $-118.8,-117.8$, and $-123.2 \mathrm{dBm}$, as shown in Fig. 8. The noise floor is about $-130 \mathrm{dBm}$ at the same frequencies, as shown in Fig. 8. The internal electric field of $29.8 \mathrm{~V} / \mathrm{m}$ in the TEM cell produces a SNR of $\sim 11.2, \sim 12.2$, and $\sim 6.8 \mathrm{~dB}$ respectively at those frequencies. Therefore, the minimum detectable electric-field are $\sim 8.21, \sim 7.24$, and $\sim 13.3 \mathrm{~V} / \mathrm{m}$, respectively at those three frequencies, based on $\mathrm{E}_{\min }=29.8 \times 10^{(-\mathrm{SNR} / 20)} \mathrm{V} / \mathrm{m}$. Figure 9 shows the sensitivity curves at frequencies of 10 , 30, and $50 \mathrm{MHz}$. We can confirm that the graph shows almost linear response for applied electric field intensity from $0.293 \mathrm{~V} / \mathrm{m}$ to $23.2 \mathrm{~V} / \mathrm{m}$, even though some data were not on the line, yet very close. The device has a dynamic range of about $\sim 10, \sim 12$, and $\sim 7 \mathrm{~dB}$ at frequencies of 10 , 30 , and $50 \mathrm{MHz}$, respectively. The measuring-frequency response of the sensor at $\mathrm{rf}$ input power of $20 \mathrm{dBm}$ rf was measured in the TEM cell, showing almost flat response from 1 to $50 \mathrm{MHz}$, as seen in Fig. 10. The high-frequency

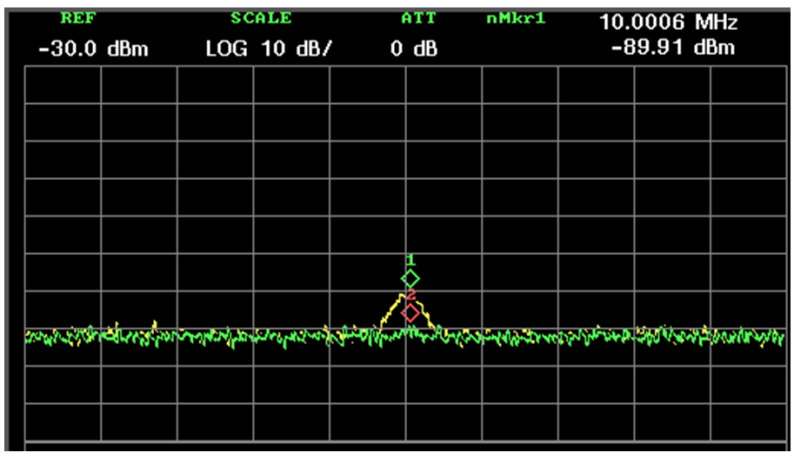

(a)

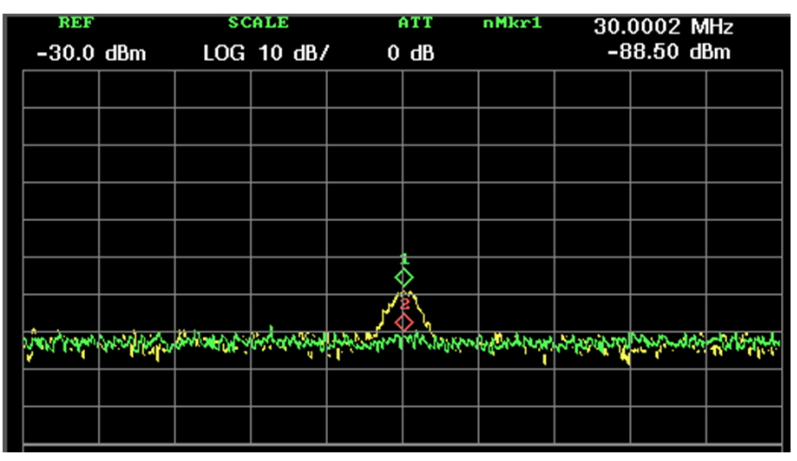

(b)

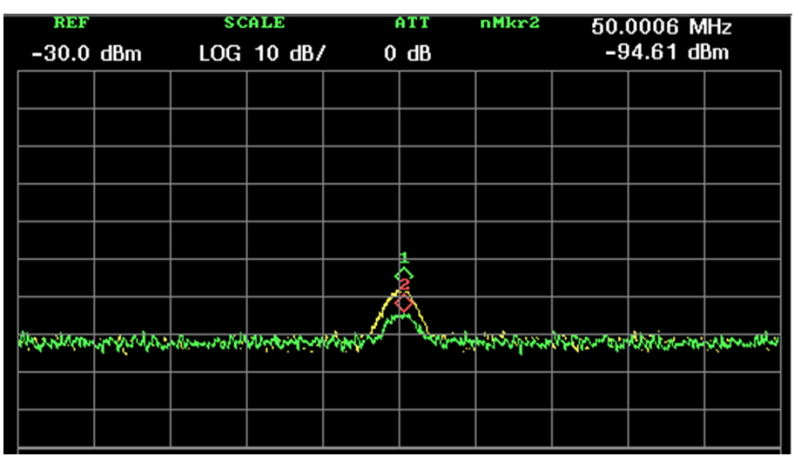

(c)

FIG. 8. The rf spectra of (a) $10 \mathrm{MHz}$, (b) $50 \mathrm{MHz}$, and (c) 70 $\mathrm{MHz}$ rf input signals into the TEM cell with a power level of $100 \mathrm{~mW}$.

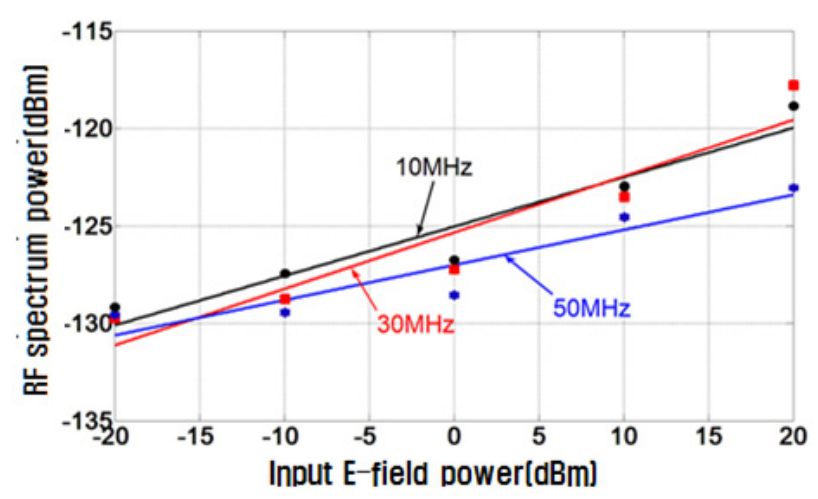

FIG. 9. Photodetected signal power versus electric-field strength into the TEM cell at different frequencies. 


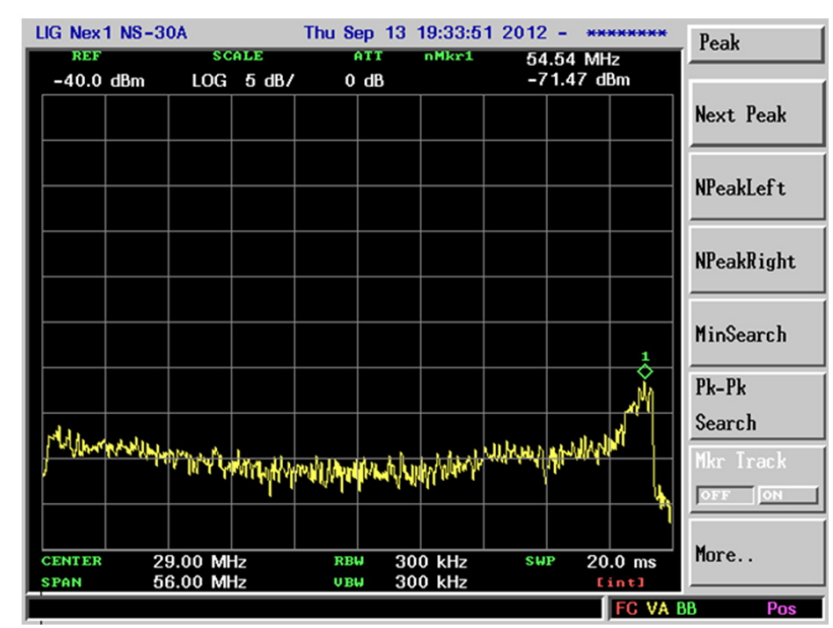

FIG. 10. The frequency response of the sensor.

cutoff results from the resistance of the electrode, which occurs in series with the modulator's capacitance. For an electrode material with higher conductivity, we can expect to obtain a higher frequency cutoff.

Theoretical analysis and experimental results show that the YBB-MZI sensor has a superior optical bias and a simple sinusoidal transfer function. For the YBB-MZI, 3-dB optical bias can be achieved when the two waveguide arms are exactly symmetrical, regardless of the refractive index of the waveguide. However, for a conventional MZI a difference in optical length of the two arms is needed to realize a bias (equal to $\pi / 2$ ). The bias depends on both the length difference and the waveguide's effective refractive index, which is affected by fabrication parameters such as titanium thickness, diffusion time, temperature, and ambient. Therefore, the YBB-MZI structure allows much better control of the optical bias than does a conventional MZI.

\section{CONCLUSION}

We have fabricated a photonic electric-field sensor that integrates a $1 \times 2$ electro-optic Ti: $\mathrm{LiNbO}_{3}$ Y-fed balanced bridge Mach-Zehnder Interferometric modulator, which provides the sensor with the unique characteristic of an intrinsic $3-\mathrm{dB}$ operating point, due to its symmetric geometry. Theoretical analysis shows that the YBB-MZI structure inherits the advantages of conventional MZI and directional coupler structures: a sinusoidal transfer function and better optical bias control. The sensor has a small device size of $49 \times$ $15 \times 1 \mathrm{~mm}$ and operates at a wavelength of $1.3 \mu \mathrm{m}$. A dc switching voltage of $\sim 25 \mathrm{~V}$ and an extinction ratio of $\sim 12.5 \mathrm{~dB}$ were observed. The minimum detectable electric fields for this device are $\sim 8.21,7.24$, and $\sim 13.3 \mathrm{~V} / \mathrm{m}$, corresponding to a dynamic range of about $\sim 10,12$, and $\sim 7 \mathrm{~dB}$ at frequencies of 10,30 , and $50 \mathrm{MHz}$ respectively. The sensor exhibits almost linear response for applied electric-field intensities from $0.29 \mathrm{~V} / \mathrm{m}$ to $29.8 \mathrm{~V} / \mathrm{m}$. In the future, we will try to improve the sensitivity, operating stability, response speed, encapsulation, and detectable frequency range. To realize high sensitivity it is necessary to improve the efficiency of the optical modulator itself, and to reduce the noise of the optical detector, as well as that of light source. Sensitivity limited by shot noise can be achieved by suppressing the influence of the relative intensity noise (RIN) of the laser diode at the optical receiver. This becomes possible using a balanced detection scheme consisting of the presented $1 \times 2$ YBB-MZI modulator in combination with a balanced detector. Because the sensitivity of electricfield sensors using various $\mathrm{Ti}: \mathrm{LiNbO}_{3}$ integrated optical modulators is also strongly affected by the structures of the electrode and antenna, we will try to apply various electrode structures and antennas (such as dipole, loop, and segmented electrode) in various integrated modulators.

\section{ACKNOWLEDGMENT}

This research was supported by the Basic Science Research Program through the national Research Foundation of Korea (NRF) funded by the Ministry of Education, Science and Technology (2010-0021491), and by the 2014 Hongik University Research Fund.

\section{REFERENCES}

1. N. Kuwabara, K. Tajima, R. Kobayashi, and F. Amemiya, "Development and analysis of electric field sensor using $\mathrm{LiNbO}_{3}$ optical modulator," IEEE Trans. Electromagn. Compat. 34, 391-396 (1992).

2. R. Zeng, B. Wang, B. Niu, and Z. Yu, "Development and application of integrated optical sensors for intense E-field measurement," Sensors 12, 11406-11434 (2012).

3. H. S. Jung, "Photonic electric-field sensor utilizing an asymmetric Ti:LiNbO 3 Mach-Zehnder interferometer with a dipole antenna," Fiber and Integrated Optics 31, 343-354 (2012).

4. T. H. Lee, F. T. Hwang, W. T. Shay, and C. T. Lee, "Electromagnetic field sensor using Mach-Zehnder waveguide modulator," Microwave and Optical Technol. Lett. 48-9, 1897-1899 (2006).

5. D. H. Naghski, J. T. Boyd, H. E. Jackson, S. Sriram, S. A. Kingsley, and J. Latess, "An integrated photonic MachZehnder interferometer with no electrodes for sensing electric fields," J. Lightwave Technol. 12, 1092-1098 (1994).

6. D. An, Z. Shi, L. Sun, J. M. Taboada, Q. Zhou, and X. $\mathrm{Lu}$, "Polymeric electro-optic modulator based on $1 \times 2 \mathrm{Y}$-fed directional coupler," Appl. Phys. Lett. 76, 98-104 (2005).

7. H. Jung, "Electro-optic electric-field sensors utilizing Ti: $\mathrm{LiNbO}_{3}$ $1 \times 2$ directional coupler with dipole antennas," Opt. Eng. 52, 064402 (2013).

8. M. M. Howerton, C. H. Bulmer, and W. K. Burns, "Linear $1 \times 2$ directional coupler for electromagnetic field detection," Appl. Phys. Lett. 52, 1850-1852 (1988).

9. R. Zeng, B. Wang, Z. Yu, B. Niu, and Y. Hua, "Integrated optical E-field sensor based on balanced Mach-Zehnder 
interferometer," Opt. Eng. 50, 114404 (2011).

10. R.-C. Twu, "Zn-diffused $1 \times 2$ balanced-bridge optical switch in a Y-cut lithium niobate," IEEE Photon. Technol. Lett. 19, 1269-1271 (2007).

11. M. Schwerdt, J. Berger, B. Schuppert, and K. Petermann, "Integrated optical E-field sensors with a balanced detection scheme," IEEE Trans. Electromagn. Compat. 39, 386-390 (1997).

12. H. Nishihara, M. Haruna, and T. Suhara, Optical Integrated Circuits (McGraw-Hill Book Company, New York, 1985), Chapter 5.

13. Optiwave, OptiBPM 9.0: Waveguide Optics Design Software. 